\title{
LAS DIDÁCTICAS ESPECÍFICAS: ENTRE LAS EPISTEMOLOGÍAS DISCIPLINARES Y LA ENSEÑANZA. ALGUNAS NOTAS SOBRE LA FORMACIÓN DEL PROFESORADO
}

\author{
Marta Susana Brovelli (Universidad Nacional de Rosario** \\ mbrovell@arnet.com.ar
}

Recibido: 28/07/2011 Aceptado: 06/12/2011

\section{Resumen}

El propósito de este trabajo es reflexionar acerca de la existencia de la Didácticas Específicas como campo de conocimientos, como un lugar construido en los bordes e intersección de la Didáctica (o Didáctica General) y de las epistemologías disciplinarias, tal como se sostiene desde hace ya muchos años en la cátedra de Área del Currículum de la carrera de Ciencias de la Educación, de la Universidad Nacional de Rosario.

Se hace referencia a dos conceptos teóricos que aportan para pensar las Didácticas Específicas: el de transposición didáctica y el de Conocimiento Didáctico del contenido (CDC), desarrollando algunas cuestiones básicas referidas a cada uno de ellos.

También se realizan algunas reflexiones acerca de las cuestiones planteadas, con referencia a su lugar y modos de trabajo en la Formación Docente, campo siempre controversial, donde aún no existen legitimaciones suficientes y firmes.

\section{Palabras clave}

Didáctica - Didácticas Específicas - Transposición Didáctica - Conocimiento didáctico del Contenido - Formación Docente.

\section{Abstract}

The purpose of this paper is to reflect upon the existence of Specific Didactics as a field of knowledge, as has been supported for many years by the class in Curriculum Area, Faculty of Education Sciences, Rosario National University.

* Doctora en Filosofía y Ciencias de la Educación - UNED (España). Especialista en Educación a Distancia - UNED (España). Profesora y Licenciada en Ciencias de la Educación (UNL). 
It proposes the existence of Specific Didactics as objects of knowledge, as a place built on the edges and the intersection of Didactics (or General Didactics) and disciplinary epistemologies.

Then it refers to two theoretical concepts provided to think Specific Didactics, they are Didactic Transposition and Didactic knowledege of content, and it develops some basic issues relating to each of them.

It also reflects upon the matter presented with reference to their place and working methods in Teacher Training, a controversial field where there are not enough or strong justifications.

\section{Key words}

Didactics - Specific Didactics - Didactic Transposition - Didactic knowledege of Content - Teacher Training.

\section{Introduccion}

Ya largos años de trabajo y de prácticas diversas en la formación de especialistas en educación y en la formación docente nos han permitido ir construyendo, con diferentes aportes teóricos de otros autores, posicionamientos acerca de algunas cuestiones básicas que abordamos sistemáticamente. Una de ellas se refiere al problema de las Didácticas Específicas y de su enseñanza.

Desde ese lugar nos permitimos partir de la siguiente afirmación: El campo teórico de la Didáctica y de las Didácticas específicas, se ha configurado desde dimensiones históricas, epistemológicas, políticas, sociales. En ellos las discusiones y debates han sido y siguen siendo moneda común. Constituyen campos en movimiento con límites imprecisos, alimentados por nuevos/viejos planteos, nuevas articulaciones conceptuales, nuevas alternativas de acción.

El tema que nos ocupa, puede ser analizado desde dos perspectivas diferentes: una de ellas es la que se puede ubicar en el campo de la reflexión y producción teórica y conceptual, mientras que la otra perspectiva tiene que ver con articulaciones entre la teoría y la práctica en problemas de diseño del curriculum, y especialmente en el de la formación docente.

Ante los planteos realizados, parece útil considerar algunos interrogantes como los siguientes:

¿A qué nos estamos refiriendo cuando hablamos de Didácticas Específicas?

¿En qué se sustenta su especificidad?

¿Cuáles son las relaciones entre Didácticas Específicas y Didáctica General o Didáctica?

¿Se encuentran estos campos conceptuales claramente delimitados y legitimados?

¿Podemos reconocer problemas y conflictos centrales en ellos? 
Partimos, además, de la existencia del campo teórico de las Didácticas Específicas (por objetos de conocimiento) como parte del campo más amplio de la Didáctica y del Currículum. Esta aseveración lleva a planteos consecuentes en cuanto a concepciones de diseño y desarrollo del curriculum y de la enseñanza, atendiendo a los objetos específicos de conocimiento como una cuestión didáctica, intentando focalizar esta cuestión en la formación de profesionales docentes.

En segundo lugar se hace referencia a dos aportes considerados muy valiosos para la construcción de las didácticas Específicas, como son el de Transposición Didáctica, de Yves Chevallard, para dedicarnos luego con mayor detenimiento al concepto de Conocimiento Didáctico del Contenido, trabajado originariamente por Shulman y luego continuado por otros investigadores y especialistas.

Finalmente se harán algunas consideraciones acerca de la formación de los docentes con respecto a la Didáctica y las Didácticas Específicas.

\section{Desarrollo y discusión}

En referencia a la primera parte de la cuestión, ubicada en el plano de la reflexión teórica y conceptual, es pertinente aclarar que no se trata de un problema nuevo, sino de una vieja cuestión que se reflota periódicamente, desde distintas perspectivas y con diferentes intereses.

Podemos afirmar que las Didácticas Específicas son parte de un campo en construcción, inacabado, que mantiene relaciones básicas e ineludibles con el de la Didáctica, en tanto a este último pertenecen conceptos, teorías y problemas de carácter general que iluminan los problemas de la enseñanza, la que se constituye en su objeto, más allá de los contenidos que se pretendan enseñar.

De esta manera, la Didáctica se ha conformado como campo disciplinar que incluye su corpus teórico, las prácticas de la enseñanza, con sus particularidades específicas, profesionales y de carácter tecnológico y la investigación de la enseñanza (Contreras, 1990). Cabe además destacar que en la actualidad la Didáctica se ha visto enriquecida con desarrollos de investigaciones cada día más abundantes, que permiten brindar sustento a consideraciones de carácter práctico para el diseño y desarrollo de la enseñanza, cuestiones que tendrán que ser tomadas en cuenta por las Didácticas Específicas para sus propias formulaciones.

Sin embargo, es también cierto que resulta imposible estudiar y analizar la enseñanza dejando de lado aquello que se pretende enseñar, y es precisamente en este punto donde aparece el requerimiento de tener en cuenta el acercamiento a otros objetos de conocimiento que pertenecen a otros campos del saber, cargados con sus propios problemas, pertenecientes a las disciplinas a las que se recurre para la selección de contenidos de la enseñanza y del aprendizaje. 
El planteo realizado es el que lleva a pensar en la necesaria convergencia para constituir las Didácticas Específicas por objeto de conocimiento, entre los conocimientos provenientes de la Didáctica y los provenientes de las distintas disciplinas específicas; se trata entonces de trabajar en la intersección entre estos campos teóricos y en las prácticas de la enseñanza que los requiere.

Si partimos de considerar que tanto la Didáctica como las Didácticas Específicas están configuradas y sostenidas desde dimensiones históricas, políticas, sociales, epistemológicas, metodológicas e institucionales, estas perspectivas de análisis deben ser tenidas en cuenta en el análisis de los avances en estos campos, como así también en las nuevas producciones que se realicen o en los debates que en torno a ello puedan surgir.

A pesar de que en muchos planes de estudio de formación del profesorado y aún en carreras de Ciencias de la Educación figuren Didácticas Especiales por nivel del Sistema Educativo, en el campo conceptual de la didáctica ya se ha rechazado claramente esta posición atendiendo a los argumentos antes desarrollados. De allí que pensemos que, en todo caso, debiéramos hablar de la Didáctica General y de las Didácticas Específicas en los distintos niveles del sistema educativo, de modo de considerarlas en situación institucional y de aula, pero siendo vistas una y otras como partes de la Didáctica.

La argumentación que sostiene lo planteado anteriormente es la siguiente: el objeto de estudio de la Didáctica es la enseñanza y sus prácticas y las correspondientes teorizaciones que sobre ella se realizan, por lo que las didácticas específicas entendidas como enseñanza de objetos pertenecientes a diferentes ciencias, se constituyen también en objetos investigación didáctica. Pregunta: ¿qué es lo que se constituye en una categoría? ¿La enseñanza? Se constituyen también en objetos de investigación didáctica.

Ahora bien, si consideramos a la Didáctica como un saber en situación, los cambios en el contexto en el que se concreta la enseñanza incluyen a las características de los sujetos del aprendizaje y de las instituciones en las que la misma se lleva a cabo. Sin embargo, lo que no cambia en el caso de contenidos pertenecientes a una disciplina determinada, es el objeto de enseñanza en cuanto a su campo de pertenencia. Esto quiere decir que se puede pensar, por ejemplo, en la enseñanza de Matemáticas o de Historia en cualquiera de los niveles del sistema educativo, y con ello estamos indicando que necesariamente cambian las características contextuales y situacionales de la enseñanza, pero el objeto de enseñanza sigue siempre perteneciendo a esos campos disciplinares mencionados.

Partiendo de la afirmación anterior, y tomando como unidad de análisis la "tríada didáctica", aquello que estaría dando la especificidad a la enseñanza es, precisamente, el objeto de enseñanza, mientras que las características del sujeto de aprendizaje estaría requiriendo del docente, como mediador entre el objeto de conocimiento y el/los sujetos, una construcción discursiva y meto- 
dológica (1) adecuada a esos sujetos, portadores de instrumentos culturales diferentes, y a las exigencias curriculares, pero respetando las características propias del objeto de enseñanza.

Esto significa que a un mismo grupo de alumnos se les pueden enseñar contenidos de distintas disciplinas: Matemáticas, Historia, Lengua, etc., y sin embargo no estamos pensando en formas de enseñanza iguales para cada una de esas clases.

Este planteo nos lleva a pensar en el centro de la tríada como lugar de convergencia de los tres vértices: docente-alumno-conocimiento; y de sus variadas interrelaciones, puede ser la ACTIVIDAD de enseñanza orientadora de los procesos de aprendizaje. La actividad sería entonces nuestra unidad de análisis, ya que a través de ellas podrían apreciarse las cuestiones más propiamente didácticas y las sucesivas adaptaciones que el docente va haciendo para lograr mejor comprensión del tema por parte de los alumnos. A través del análisis de las actividades que propone el docente es posible apreciar si intenta el desarrollo del tema y su comprensión, por parte de los alumnos, tanto los datos pertinentes como los procedimientos requeridos para su mejor aprendizaje.

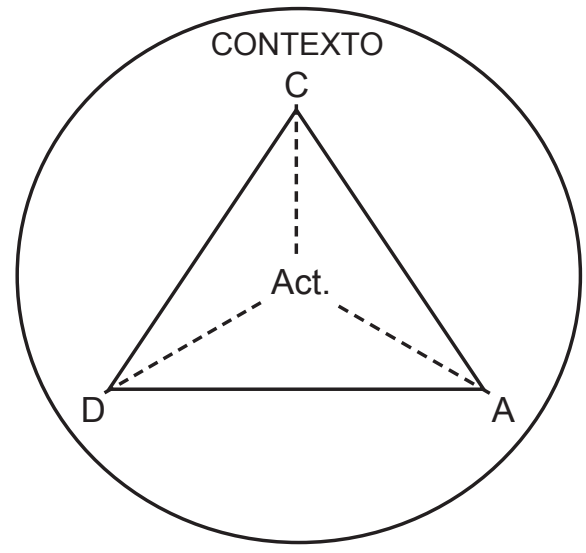

Decimos entonces que para poder pensar formas de enseñanza adecuadas, de contenidos pertenecientes a diferentes disciplinas científicas es necesario, desde el punto de vista epistemológico, tener en claro la estructura sintáctica y la estructura semántica de las diferentes disciplinas, así como su construcción y su devenir histórico. Sólo el manejo de estos aspectos de las disciplinas nos puede asegurar una selección, organización y desarrollo adecuado de los contenidos correspondientes.

Sin embargo, la Didáctica nos dice que estos aspectos disciplinares mencionados no cubren los conocimientos necesarios para abarcar el análisis y la 
comprensión de la complejidad de la clase y resultan insuficientes a la hora de poner la clase en acción.

Es precisamente aquí, en este punto, donde las Didácticas Específicas suelen presentar un vacío conceptual y práctico que justifica la necesaria recurrencia a la Didáctica, en tanto es en éste campo donde se pueden encontrar conocimientos e investigaciones que nos permitan pensar la clase, como espacio en el que se concreta la enseñanza, desde distintas perspectivas tratando de considerar su complejidad y la posibilidad de decisiones metodológicas adecuadas a ella, a través de procesos de reflexión y acción.

Queremos aquí, a modo de alerta, señalar una situación de riesgo en la que a nuestro criterio están cayendo algunas experiencias y formulaciones conceptuales y técnicas o tecnológicas que se están produciendo en el espacio de las Didácticas Específicas.

Nos referimos concretamente a la influencia innegable y valiosa, pero insuficiente, de la psicología y más precisamente de la psicología cognitiva. Esto está llevando a construir propuestas didácticas sesgadas, estructuradas y poco fiables en sus posibles resultados en tanto no atienden a otras condiciones contextuales y dinámicas de las situaciones de clase.

Esto último es lo que nos lleva a sostener que el campo de las Didácticas Específicas es un campo de construcción compartido, en diálogo permanente, entre los especialistas en Didácticas Específicas y los especialistas en Didáctica, evitando de esta manera fragmentaciones inútiles y, más aún, perjudiciales, en tanto en realidad, son partes de un todo que es la Didáctica.

Desde este planteo es que señalamos la importancia de generar espacios de trabajo cooperativo entre los especialistas mencionados, en tanto es difícil, aunque no imposible, encontrar especialistas que reúnan en sí mismos, ambos tipos de conocimientos: los disciplinares y los didáctico-curriculares, en su doble carácter de saberes teóricos y prácticos.

Estos espacios tienen que lograrse tanto a nivel de la formación de los docentes como en la construcción de diseños curriculares (macro y micro) y consecuentemente tienen que ser también espacios de debate y construcción teórica.

En este espacio en concreto que hoy se nos ofrece pretendemos también reflexionar acerca de distintos aspectos que puedan conducir a una dialéctica entre profesores de "disciplinas específicas" con profesores de "didácticas de disciplinas específicas", con, a su vez, profesores de "didáctica general" y otros espacios de formación pedagógica.

Siguiendo a Carlos Marcelo García (1993), podemos plantearnos cuestiones como las siguientes: ¿qué tipo de especialización didáctica han de recibir los aspirantes a profesores? ¿Qué modelo de profesional de la enseñanza buscamos? ¿Qué rol le cabe a la Universidad en esta búsqueda? En otras palabras: ¿cuáles son los conocimientos, destrezas, actitudes, disposiciones 
que ha de poseer un profesor, en los tiempos que corren, para satisfacer la demanda educativa de América Latina?

Seguramente las respuestas a estas preguntas nos puedan esclarecer algunas dudas respecto al proceso de elaboración de planes de estudio en todos los niveles de la educación y, en particular, en el profesorado. No escapa a este análisis que cada cual está convencido de la importancia que tiene para la formación del profesorado la disciplina en la que es especialista, así como los conocimientos que se producen en las Universidades $Y$ en ello se centrará la atención.

En la actualidad nos encontramos ante un nuevo proceso de cambios en la formación de los docentes. Ello indefectiblemente nos lleva a tener en cuenta cuestiones como las siguientes:

- Toda reforma en la formación del profesorado implica plantearse qué modelo de profesional de la enseñanza desea formar.

- Este modelo de profesional ha de especificarse en relación a qué conocimientos, destrezas y actitudes ha de poseer el docente de los diferentes ámbitos disciplinares

- El conocimiento del profesor se construye en interacción con el ambiente de aprendizaje que la formación del profesorado ofrece.

- Existen diferentes resultados de investigaciones acerca del conocimiento profesional de los profesores que es necesario conocer para poder desarrollarlos en el profesor en formación.

- Las diferentes disciplinas o especialidades incorporan unas estructuras de conocimiento específicas que han de ser conocidas por los profesores formados y en formación.

- El conocimiento sobre el contenido de una disciplina es necesario pero insuficiente para una enseñanza que busque que los estudiantes comprendan, asimilen y elaboren.

- $\quad$ Es preciso prestar mayor atención -conceptual y empírica- a la forma en que los profesores "transforman" el conocimiento que poseen de la materia en conocimiento "enseñable" y comprensible para otros estudiantes.

\section{Aportes significativos para la construcción de las didácticas específicas.}

En el camino por el logro de reconocimiento epistemológico en la construcción del campo propio de las Didácticas Específicas, pueden identificarse dos conceptos importantes provenientes de teorizaciones e investigaciones, que si bien presentan algunas diferencias entre sí y también algunas limitaciones, constituyen aportes valiosos.

Se trata de la llamada "Transposición didáctica" de Yves Chevallard, y el concepto de "Conocimiento Pedagógico del Contenido", denominado así 
por Shulman $(1986 / 7,1990,1999,2001)$, a partir de una amplio proyecto de investigación que él inicia y dirige en USA, concepto que Bolívar (1993,2005) pasa a denominar como "Conocimiento didáctico del contenido", forma que considera más apropiada según la tradición europea de la Didáctica. A ellas haremos referencias básicas.

\section{La transposición didáctica}

Hace ya bastante tiempo que hemos podido tomar, ir incorporando y trabajando en la formación de los docentes y de los especialistas en Ciencias de la Educación, el concepto de transposición didáctica de Yves Chevallard (1991). Este autor construye el concepto y teoriza en torno a él, a partir de su experiencia y reflexión acerca de la enseñanza de la Matemática. Sin embargo, por su valor de orden general para la enseñanza de cualquier contenido específico, integra ya el bagaje de la Didáctica. El planteo central es el que permite afirmar que el conocimiento a ser enseñado pasa por cambios sucesivos hasta que se convierte en conocimiento enseñado. Estos cambios, propios de la situación didáctica, de la enseñanza no tienen que ver sólo con las características propias del contenido a ser enseñado sino que se relacionan con cuestiones curriculares, institucionales y propias del ámbito de la enseñanza, que es el aula. Las sucesivas reformulaciones, mediaciones y atención a aspectos referidos a la intersubjetividad y al carácter político y social de la enseñanza son cuestiones a ser tenidas en cuenta cualquiera sea el contenido a ser enseñado y enseñado, ya que el objeto de la Didáctica, la enseñanza, es un objeto en situación y por lo tanto, el saber que se construya acerca de ella así debe considerarlo. Como afirmaba el propio Chevallard (1991), "hay transposición didáctica porque el funcionamiento didáctico del saber es otro que el funcionamiento académico".

De este modo, plantea Chevallard (1991, p. 41) que:

un contenido de saber que ha sido designado como saber a enseñar, sufre (...) un conjunto de transformaciones adaptativas que van a hacerlo apto para ocupar un lugar entre los objetos de enseñanza.

El 'trabajo' que transforma de un objeto de saber a enseñar en un objeto de enseñanza, es denominado la transposición didáctica.

¿Cómo los saberes científicos son "transpuestos" a contenidos educativos? El fenómeno de la transposición didáctica comprende, pues, las sucesivas transformaciones, contextualizaciones o desplazamientos que se producen en el conocimiento, desde que es elaborado por la comunidad científica hasta su vehiculización institucionalizada como conocimiento escolar. Esto le permite platear una alerta muy importante para la enseñanza como lo es el llamado a la "necesaria vigilancia epistemológica".

Además de esta reactualización del "triángulo didáctico", Chevallard elabora una concepción más compleja o sistémica, ubicando al sistema educativo en 
lo que el llama la noosfera, que le permite situar el saber y el sistema de prácticas en un contexto más amplio. La noción de institución le permite inscribir la situación didáctica en las prácticas sociales compartidas en que tienen lugar. De este modo, el aprendizaje se juega en la adecuación entre los significados personales e institucionales en que ocurren.

\section{Conocimiento didáctico del contenido}

Nos detendremos algo más en este concepto, pues a nuestro entender es menos conocido y trabajado en el contexto de nuestro país. Desde hace ya algunas décadas numerosos estudios comenzaron a interesarse por indagar acerca del conocimiento de los profesores, más específicamente sobre el Conocimiento Didáctico del Contenido, refiriéndose a aquellos estudios en los que se analiza específicamente el conocimiento que los profesores poseen respecto al contenido que enseñan, así como -y esto es fundamental-, la forma como los profesores trasladan ese conocimiento a un tipo de enseñanza que produzca comprensión en los estudiantes. Como se puede observar, el cambio que se viene produciendo en la investigación pensada en sus orígenes como de "Pensamientos del Profesor" es hacia una investigación más vinculada con los contenidos que enseñan los profesores.

A este respecto, Elmore (1992) plantea que

Es probable que la enseñanza eficaz varíe considerablemente de disciplina en disciplina. A diferencia de la investigación sobre enseñanza eficaz, que intentaba identificar destrezas docentes genéricas, la actual investigación se centra en las relaciones entre la enseñanza y el aprendizaje en disciplinas específicas. Aunque es posible extraer semejanzas entre los diferentes tipos de destrezas que se requieren a lo largo de las diferentes áreas de contenido, la actual investigación sobre la enseñanza se centra principalmente en los requisitos específicos para comprender una disciplina" (p. 45).

Y ésta es una de las aportaciones más relevantes de las actuales investigaciones que están tratando de comprender la complejidad de la enseñanza de las diferentes disciplinas académicas que configuran el curriculum escolar.

\section{El Conocimiento Didáctico del Contenido como un tipo especial de conocimiento del profesor}

En términos generales es común que se reconozca que los profesores deben tener al menos tres tipos de conocimientos diferentes, que se ponen en juego en la enseñanza: los profesores han de conocer fundamentos pedagógicos, didácticos y psicológicos desde un nivel genérico, es decir sin especialización disciplinar; en segundo lugar, los profesores han de poseer un conocimiento del contenido que enseñan, comprendiendo tanto su estructura 
sustantiva como sintáctica. En tercer lugar, los profesores han de conocer cuál es su audiencia, cuáles son las características de los estudiantes a los que van a enseñar el contenido que conocen.

Sin embargo, sabemos también, que la posesión de esos tres tipos de conocimiento no asegura ni son base suficiente para producir buenas propuestas de enseñanza. ¿Qué será entonces lo falta, lo que parece estar ausente? Podríamos decir que en esta secuencia está ausente un componente fundamental que es precisamente los que se ha llamado como Conocimiento Didáctico del Contenido.

Sabemos los profesores no enseñan la materia a sus estudiantes (al menos en los niveles no universitarios) tal como la han estudiado o tal como la conocen como especialistas en una disciplina. Los profesores, bien de forma consciente o inconsciente adaptan, reconstruyen, reordenan, y simplifican el contenido para hacerlo comprensible a los estudiantes ¿Cómo se produce este proceso?, ¿en qué medida afecta el nivel de comprensión que un profesor tenga de una disciplina a la calidad de esta 'transformación'?, ¿en qué medida la formación inicial del profesorado contribuye a facilitar el desarrollo de estos procesos de transformación?, ¿qué diferencias existen en estos procesos según las diferentes disciplinas? Algunas de estas preguntas están empezando a tener respuesta gracias a la investigación que se ha venido desarrollando en los últimos siete años sobre el Conocimiento Didáctico del Contenido.

En la revisión que realizara Shulman en 1989 en torno a los programas de investigación didáctica ya se apuntaba que "faltaba" en ellos algo:

donde el programa sobre el pensamiento del profesor ha fallado evidentemente es en la dilucidación de la comprensión cognitiva del contenido de la enseñanza por parte de los enseñantes; y las relaciones entre esta comprensión y la enseñanza que los profesores proporcionan a los alumnos (Shulman, 1989, p. 65).

En definitiva, Shulman plantea que la investigación didáctica ha prestado poca atención a cómo se enseña y qué dificultades tiene la enseñanza de cada una de las disciplinas o áreas que componen el curriculum escolar.

En esta línea se ha venido trabajando en diferentes contextos educativos para ir apuntando respuestas a algunas de las preguntas anteriormente enunciadas. Por Conocimiento Didáctico del Contenido, Shulman expresa lo siguiente:

Dentro de la categoría conocimiento didáctico del contenido incluyo los temas más comúnmente enseñados en una determinada asignatura, las formas más útiles para representar las ideas, las analogías, ilustraciones, ejemplos, explicaciones y demostraciones más poderosas, en una palabra, las formas de representar y formular el 
contenido para hacerlo comprensible a otros. El conocimiento didáctico del contenido también incluye un conocimiento de lo que facilita o dificulta el aprendizaje de temas concretos; las concepciones y preconcepciones que los estudiantes de diferentes edades y procedencia traen consigo cuando aprenden los temas y lecciones más frecuentemente enseñadas (Shulman, 1986, p. 9-10).

Más recientemente, Stengel, afirmaba que

el profesor inevitablemente transforma el contenido en algo, un contenido enseñable que tiene su propia lógica y estructura, y tiene sentido para los alumnos. El conocimiento que ayuda a que se produzca esta transformación del conocimiento incluido en el curriculum escolar, en algo que tenga sentido para los alumnos, es a lo que denominamos "Conocimiento Didáctico del Contenido (Stengel, 1992, p. 7, citado por García C.M.).

Este tipo de conocimiento es el que permite transformar el conocimiento original en conocimiento que pueda ser incorporado por los estudiantes teniendo en cuenta sus conocimientos previos, así como sus posibles errores conceptuales.

Podríamos hasta aquí decir entonces que el Conocimiento Didáctico del Contenido se construye a partir del conocimiento del contenido que el profesor posee, así como del conocimiento pedagógico general, del conocimiento de los alumnos, y también es consecuencia de la propia biografía personal y profesional del profesor.

Si nos referimos en especial al conocimiento del contenido a enseñar, se hace necesario aclarar cómo éste se va conformando. Al respecto podemos afirmar que, en primer lugar, el conocimiento del contenido curricular tiene a su vez, distintas dimensiones que deben ser enseñadas y aprendidas: el conocimiento del contenido de la materia (hechos, conceptos centrales o principios organizativos), conocimiento substantivo (marcos explicativos de la disciplina), conocimiento sintáctico, y creencias sobre la materia. Ante ello nos preguntamos si en la formación de los profesores se asegura que el conocimiento del contenido disciplinar realmente incluya lo mencionado anteriormente. Pero más aún, sabemos que conocer un contenido no implica saber enseñarlo, por eso es posible afirmar que el conocimiento del contenido es una condición necesaria, aunque no suficiente para una buena enseñanza, a ello es necesario agregar la categoría de "conocimiento didáctico del contenido", como capacidad del profesor para entender las formas alternativas del currículum de su respectivo campo disciplinar, presentarlo a los alumnos y de discutir los modos en que este contenido está expresado en los materiales y textos.

El "conocimiento didáctico del contenido", tal como afirma Bolívar (2005), tiene un estatus propio y es más que la conjunción o intersección entre el conoci- 
miento de la materia per se y los principios generales didácticos y pedagógicos. Es la capacidad para trasladar/transformar el conocimiento de la materia en representaciones didácticas (significativas, comprensibles o asimilables) para los alumnos. De este modo, si el "buen" profesional de Secundaria es aquel que no sólo tiene un conocimiento del contenido del campo disciplinar, sino también el que tiene un "conocimiento didáctico" de dicho contenido, sería función de las didácticas específicas - en conjunción con la Didáctica General - proporcionar dicho conocimiento.

El Conocimiento Didáctico del Contenido permite explicar mejor algunas de las fases que median entre la planificación y la enseñanza (García, 1987b). En la investigación sobre el pensamiento del profesor se ha venido asumiendo la distinción hecha por Jackson (1975) al identificar las fases pre, inter y postactiva, en el proceso de enseñar. Sin embargo, Shulman (1987) ha planteado que, partiendo de esas fases, conviene especificar otros procesos que se producen y que tienen que ver fundamentalmente con la transformación del contenido en materia enseñable.

Todo proceso de enseñanza comienza con una reflexión y elaboración (conocimiento comprensivo) de los propósitos, estructura del contenido a desarrollar, de las ideas y relaciones que es posible desarrollar dentro de la propia materia y con otras disciplinas. Paralelamente, los profesores comienzan la transformación del contenido que van a desarrollar incluyendo una selección y organización de los materiales a utilizar; la selección de analogías, metáforas, ejemplos, demostraciones, explicaciones, etc., para adaptar el contenido a las características de los estudiantes, teniendo en cuenta las concepciones, preconcepciones, errores conceptuales, dificultades, lenguaje, cultura, motivaciones, clase social, sexo, edad, capacidad, aptitud, intereses, autoconceptos, y atención de los alumnos. Esta es una fase de especial importancia y a la que se le ha dedicado escasa atención. El tercer componente identificado por Shulman es la enseñanza, e incluye conocimiento referido a la gestión de clase, presentación de la información, interacción con los alumnos, trabajo en grupo, disciplina, etc. Paralelamente a la enseñanza, los profesores procesan información, a través de la cual evalúan la comprensión de los estudiantes durante la enseñanza, así como reflexionan sobre su propia actuación. Con posterioridad a la enseñanza interactiva se produce la reflexión en la que el profesor revisa, reconstruye, analiza críticamente su propia actuación en clase.

El modelo elaborado por Shulman tiene en cuenta las propuestas de Schön (1983) sobre la epistemología de la práctica cuando plantea que los profesionales, incluidos los profesores aprenden a partir de la reflexión-enla-acción. La reflexión en este modelo no aparece en una sola fase sino que está permanente en todas las fases del proceso de enseñar. Lo original del modelo propuesto por Shulman reside en destacar la importancia de la fase de Transformación del contenido incluido en las propuestas curriculares, libros 
de texto, etc., en conocimiento enseñable. Quizás sea este el elemento en el cual se requieran más trabajos de investigación en la medida en que poco se conoce acerca de cómo se lleva a cabo y cuáles son las repercusiones en la enseñanza. Pensamos que en la formación de los profesores sería muy positivo trabajar las posibles transformaciones del contenido para enseñarlo como forma de ir construyendo un bagaje de alternativas para la enseñanza de diferentes contenidos específicos, siempre y cuando las propuestas puedan ser fundamentadas y no simplemente como alternativas de tipo técnico, aplicables a en cualquier contexto de enseñanza y de aprendizaje.

Tal como ya dijéramos, el CDC, pues, es una subcategoría del conocimiento del contenido e incluye diversos componentes: "los tópicos que más regularmente se enseñan en un área, las formas más útiles de representación de las ideas, las analogías más poderosas, ilustraciones, ejemplos, explicaciones y demostraciones, y, en una palabra, la forma de representar y formular la materia para hacerla comprensible a otros" (Shulman, 1989, p. 9).

El contenido de orientación disciplinar ha de ser reorganizado y transformado, teniendo en cuenta los alumnos, el contexto y el currículum. Una parte importante de esta recontextualización consiste en encontrar relaciones y posibilidades nuevas entre el contenido y su representación, fruto de un largo proceso en los profesores veteranos, que disponen a menudo de modelos altamente elaborados para enseñar su materia; incluyendo una "comprensión" de lo que significa la enseñanza de un tópico particular y de los principios, técnicas y modos de representar y formular la materia didácticamente.

Como atributo del conocimiento que poseen los "buenos" profesores con experiencia, el CDC se configura como una mezcla de contenido y didáctica, en que "además del conocimiento per se de la materia incluye la dimensión del conocimiento para la enseñanza". Estos profesores tienen un modelo flexible del contenido pedagógico, que - con implicaciones epistemológicas y éticas - determina tanto su desarrollo curricular práctico como la legitimación de las estrategias didácticas empleadas/excluidas. El manejo del CDC es el que permitiría a los profesores utilizar diferentes modos de enseñaza para los diversos temas o contenidos de una materia, pudiendo de esta obtener un mayor potencial de las propuestas curriculares.

Este CDC, frecuentemente puede entrar en contradicción con el que viene expresado en los libros de texto. En su trabajo cotidiano el profesor con CDC, como agente de desarrollo curricular, establece una relación entre su conocimiento, el expresado en el texto escolar y el contexto de su clase. Recrear o reconstruir el contenido de acuerdo con las perspectivas propias y el contexto de la clase, convirtiéndolo en "enseñanza" sería realizar el CDC.

El objeto, pues, de las didácticas específicas, desde la perspectiva del equipo de Shulman de Stanford, es investigar las diferentes estrategias de transformación de los contenidos de enseñanza en modos que puedan ser 
potentes didácticamente, según la materia de que se trate y el alumnado a que se dirija. Shulman argumenta que

"la enseñanza y su efectividad funciona diferentemente en los contenidos de las diferentes áreas. La enseñanza de las matemáticas a niños es dramáticamente diferente de la enseñanza de la literatura a adolescentes o adultos. Un conjunto de principios didácticos serían inadecuados para recoger conjuntamente la enseñanza de la física y la enseñanza de la historia. Los contenidos de la enseñanza precisan ser incluidos como un aspecto central de los estudios sobre la enseñanza" (Shulman y Quinlan, 1996, p. 409).

Así, en su estudio de casos sobre comparación de profesores expertos y noveles, Gudmundsdottir (Gudmundsdottir y Shulman, 1990) ejemplifica cómo, aunque los dos profesores son expertos en los conocimientos de sus respectivas disciplinas, el profesor veterano tiene un punto de vista comprehensivo y flexible de la materia, lo que le permite transformar el contenido disciplinar en "Conocimiento Didáctico del Contenido" y redefinir el Conocimiento de la Materia. El profesor con Conocimiento Didáctico del Contenido ha desarrollado la capacidad de poder recombinar, utilizar y desarrollar de diversos modos el potencial del currículum, viendo los pros y contras de cada estrategia, mientras el novel conoce un desarrollo lineal, que es el que imperturbablemente sigue en un tiempo dado, sin tener una visión global que le permita conectar y recombinar los elementos. Esto sugiere que "visualizar unidades más extensas en términos del currículum es un elemento importante del CDC", pudiendo formar más potentes y extensas estructuras que organicen las diferentes unidades discretas de información. El novel, por el contrario, sólo puede usar pequeñas piezas de información, sin organizarlas en estructuras coherentes. Como concluyen Gudmundsdottir y Shulman (1990, p. 33):

La implicación para la formación del profesorado es que ésta debe centrarse más en el Conocimiento Didáctico del Contenido. Actualmente, en la mayoría de los programas de formación del profesorado, los estudiantes aprenden primero la materia, métodos generales de enseñanza, psicología y sociología. Pero se hace poco énfasis en conseguir que los profesores en formación piensen sobre la materia que han de enseñar en términos de sus contenidos didácticos. Los profesores en formación necesitan ser conscientes del proceso que deben emprender para hacer que el conocimiento del contenido sea asequible para los alumnos, (...) para que comiencen a redefinir su Conocimiento de la Materia y, por tanto, a construir su Conocimiento Didáctico del Contenido.

La propuesta de Shulman constituye una buena base para que las didácticas específicas adquirieran una identidad epistemológica y -sobre todo- por 
su potencialidad para generar una investigación útil para la formación del profesorado de Secundaria.

\section{Aportes del concepto de Conocimiento Didáctico del Contenido para las Didácticas Específicas.}

Nos encontramos ante una línea de investigación con una historia reciente, por lo tanto los resultados pueden considerarse como tentativos, provisionales y derivados de investigaciones en las que el estudio de caso ha sido la estrategia preferida por los investigadores.

Las investigaciones realizadas hasta el momento referidas a los conocimientos del profesor acerca de la enseñanza y más específicamente del conocimiento didáctico del contenido, permiten afirmar que:

1. los profesores mantienen concepciones, creencias, orientaciones acerca de la materia que enseñan, y de cómo enseñarla. Estas concepciones están originadas en el conocimiento que el profesor posee de la materia, pero también en las experiencias no formales, de la vida cotidiana.

2. En segundo lugar también se puede afirmar que los profesores utilizan representaciones variadas para hacer comprender a los alumnos los contenidos curriculares, y que estas representaciones se diferencian en calidad y riqueza en función del conocimiento de la materia que los profesores posean, así como de la experiencia docente.

3. En tercer lugar, y en relación con lo anterior, cabe afirmar que se han establecido conexiones estables entre el Conocimiento Didáctico del Contenido de los profesores y su enseñanza interactiva. Asimismo, se puede afirmar también que se han establecido conexiones, aun poco estables, entre el Conocimiento Didáctico del Contenido y el aprendizaje de los alumnos.

\section{Aportes para la formación docente}

El Conocimiento Didáctico del Contenido no se origina por primera vez cuando el profesor en formación comienza su periodo formativo. Los estudiantes de los profesorados han sido también alumnos que han debido asimilar unos conocimientos en su historia escolar a través de los distintos profesores que en ella han tenido y que les han enseñado de diversas maneras. Estos profesores en formación han aprendido no sólo el contenido académico, sino también algunas formas de representación de ese contenido utilizadas por sus propios profesores.

Es cierto que este "aprendizaje de observación", no es un aprendizaje consciente y explícito. En el caso de los profesores de educación secundaria, el aprendizaje de observación se prolonga al menos los cinco años que duran sus 
estudios de profesorado. En estos años, estos estudiantes aprenden también los contenidos de la disciplina de su especialidad (tanto su estructura sintáctica como sustantiva), pero seguramente también desarrollan un conocimiento tácito acerca de cuáles son los mejores métodos para enseñar esa disciplina, cuáles son las partes más fáciles y difíciles, etc.

\section{Algunas conclusiones}

Si asumimos, como hipótesis, que el Conocimiento Didáctico de/ Contenido es el tipo de conocimiento específico de la profesión docente, imprescindible para desarrollar una enseñanza que propicie la comprensión y la construcción de conocimientos por parte de los alumnos, la consecuencia evidente es que este tipo de conocimiento ha de ser el eje estructurador de los programas de formación del profesorado. Sin embargo, el análisis del los currícula de la formación del profesorado y de las prácticas a través de las que se concreta, nos permite afirmar que la enseñanza de los contenidos específicos y los de las materias llamadas "pedagógicas" transcurren por carriles separados, que raras veces intentan cruzarse, situación que se ve con más claridad en la formación de los profesores para la enseñanza secundaria.

Nos preguntamos entonces ¿Cuándo se integran? ¿Cómo se integran? ¿Se enseña a integrarlos o se espera que lo hagan los estudiantes por sí mismos? ¿Será acaso por ensayo y error? Encontrar respuestas positivas a estas cuestiones es una tarea primordial de la formación del profesorado.

El hecho de que en las carreras de Formación Docente Universitarias existan Áreas de Conocimiento de Didácticas Específicas no es un criterio que de por sí asegure que los estudiantes adquieren un adecuado Conocimiento Didáctico del Contenido. Se hace necesario revisar los contenidos (conocimientos, destrezas, actitudes) básicos para la formación del profesor.

Antes de finalizar estas referencias al Conocimiento Didáctico el Contenido y también a las realizadas en cuanto a la Transposición didáctica, como aportes para la construcción de las Didácticas Específicas, consideramos oportuno decir que, si bien reconocemos el valor de ambos, tenemos también que decir que no son suficientes, al menos por dos cuestiones:

- La concepción de profesionalidad que sostienen es a nuestro criterio restringida, en tanto y en cuanto nosotros aspiramos a la formación de profesionales como intelectuales transformadores y para ello es necesario avanzar un poco más allá del conocimiento didáctico, para poder ubicar a la enseñanza en su dimensión política, histórica y ética.

- Ambos conceptos ampliamente trabajados por sus autores y seguidores, no discuten ni hacen referencia a los problemas intrínsecos en la generación y construcción de los saberes científicos y saberes como contenidos curriculares, por lo que parecen partir del conocimiento a ser enseñado o el conocimiento del contenido curricular sin problematizarlo. Se obvia 
de este modo, la perspectiva histórica, política y social del surgimiento y desarrollo de los conocimientos y saberes científicos.

Nos permitimos agregar a las dos cuestiones anteriores, como condición necesaria para producir y construir buenas propuestas de enseñanza, la necesidad de que el docente pueda reflexionar acerca de sus propias relaciones con el conocimiento, en tanto y en cuanto, según sean ellas, así serán los modos de enseñarlo que pueda e ir logrando en su trabajo profesional.

\section{Caminos iniciados y posibles recorridos}

Es posible que consideraciones como las realizadas, nuevos resultados de investigaciones y oportunas y adecuadas reformulaciones curriculares y de la enseñanza, vayan permitiendo superar la actual disociación entre la formación específica y la formación pedagógica, un viejo problema cuya persistencia no deja de pre-ocuparnos. Esta situación se ve agravada en los profesorados de enseñanza media en los que tiene lugar una clara desvalorización de la formación pedagógica frente a la específica o, como suele llamársela científica. Ante ello resulta inevitable preguntarme algunas cosas como las siguientes:

Si los profesores de enseñanza media valorizan muy positivamente su formación específica a la que por otro lado, se le dedica el mayor tiempo en la formación de grado ¿cómo es posible que desde la perspectiva social y aún de la profesional debamos reconocer el bajo nivel de aprendizaje de los alumnos? ¿qué está sucediendo con la enseñanza en los niveles obligatorios del sistema educativo? ¿Qué es realmente lo que aprenden de su disciplina los profesores? ¿cómo lo transforman en contenidos de enseñanza de modo de producir buenos aprendizajes? ¿dónde están los vacíos de saber y de poder?

Con respecto a la en general desvalorizada formación docente:

¿Por qué la minusvaloran si es aquella que tiene que ofrecer las notas específicas en cuanto al rol profesional elegido: el ser docentes?

¿Qué tipo de formación docente se brinda y que características son las que hacen a su desvalorización?

¿Esta desvalorización es una cuestión apriorística o surge de cuestiones sentidas como reales durante la formación de grado?

Es probable también, que nuevos aportes como el que estuvimos viendo referido al CDC puedan contribuir a mejorar la formación y las prácticas profesionales, reconociendo también sus limitaciones. Ahora bien, Marcelo García se pregunta: ¿quién es el guardián del Conocimiento Didáctico del Contenido?, o dicho de otra forma ¿quién enseña a los profesores el Conocimiento Didáctico del Contenido? Según este autor la respuesta es: nadie. Nadie y todos. Tal como se ha podido ir vislumbrando a lo largo de esta exposición de ideas y conceptos, el Conocimiento Didáctico del Contenido se configura a través de 
las interrelaciones entre formadores de profesores especialistas en contenidos, y especialistas en didáctica, así como en didácticas específicas, y profesores en ejercicio.

Se hace necesario, por tanto, plantear proyectos de formación y de investigación en los cuales participen a nivel de igualdad profesores de las diferentes características enunciadas anteriormente, que aúnen sus diferentes concepciones y conocimientos para contribuir a configurar un curriculum homogéneo en la formación del profesorado, que propicie la adquisición de un conocimiento integrado e interrelacionado, que plantee las dificultades y dilemas de la práctica de la enseñanza.

La segunda idea que desarrolla este autor (Marcelo García) y que compartimos, es la de plantear desde una nueva perspectiva las relaciones entre el Curriculum y la Didáctica como campos de conocimiento e investigación. La investigación didáctica, hasta hace poco tiempo se ha caracterizado por desarrollar un discurso generalista, sin especificaciones en contenidos concretos. Paralelamente, algunas perspectivas de estudios curriculares lo han entendido como el estudio de los contenidos que se enseñaban en la escuela, sin atender a su concreción didáctica. Recientemente ha planteado Doyle (1992) que la intersección entre el curriculum y la didáctica hay que buscarla en la transformación que se produce a nivel de escuela en primer lugar, y a nivel de clase en segundo lugar, del contenido especificado en el curriculum, entendiendo que "el curriculum no es simplemente el contenido, sino una teoría del contenido, es decir, una concepción de lo que significa el contenido, de lo que significa conocer ese contenido, y de qué se pretende conseguir cuando se está enseñando un contenido" (Doyle, 1992, p. 507). La investigación sobre cómo se produce esta transformación en las diferentes áreas de especialidad curricular puede ofrecernos información de gran utilidad para replantear nuestros cursos de formación didáctica en la formación inicial del profesorado. Este sería un paso hacia delante en la investigación y aportes para la construcción de propuestas en los puntos de intersección entre la Didáctica y el Currículum.

\section{Notas Bibliográficas}

(1) Brovelli, M. (2007) "La formación docente y la investigación en Didácticas Específicas”. En Cuadernos del CEDE, UNSAM, Buenos Aires.

\section{Referencias bibliográficas}

- Benejan, P. y Pages, J. (Coord.) Comes, Pilar; Quinquer, Dolores (1997). Enseñar y aprender ciencias sociales, geografía e historia en la educación secundaria. Barcelona: ICE- Horsori.

- Bolívar, A. (1993). Conocimiento didáctico del contenido y formación del profesorado: El Programa de L. Shulman. Revista Interuniversitaria de Formación del Profesorado, 16 (enero-abril), 113-124. 
- Bolívar, A. (1995). El conocimiento de la enseñanza. Epistemología de la investigación curricular. Granada: Force/Universidad de Granada.

- Bolívar, A. (2004). El conocimiento de la enseñanza: explicar, comprender y transformar. Revista Mimesis-Ciências Humanas (Bauru-Sao Paulo), vol. Profesorado. Revista de currículum y formación del profesorado, 9, 2 (2005) 3525 (1), 17-42. Disponible en: http://www.edusc.com.br/colecoes/revistas/mimesis/index.htm

- $\quad$ Chevallard, Y. (1991). La transposición didáctica. Del saber sabio al saber enseñado. Buenos Aires: Ed. Aique. Nueva edición ampliada de la original de 1985.

- Doyle, W. (1985) "La investigación sobre el contexto del aula: Hacia un conocimiento básico para la práctica y la política de formación del profesorado". Revista de Educación, 277 (mayo-agosto), 29-42.

- $\quad$ Escudero, J.M. (1993). La construcción problemática de los contenidos de la formación de los profesores. En Montero Mesa, L. y Vez, J.M. (eds.), Las Didácticas Específicas en la Formación del Profesorado (I). Santiago de Compostela: Tórculo, 71-91. -43. http:// www.ugr.es/local/recfpro/Rev92ART6.pdf 36

- Galindo, R. (1998). La didáctica de las ciencias sociales desde la perspectiva de la historia de Shulman sobre el conocimiento didáctico del contenido: una propuesta para la formación inicial Iber, n. 18, 85-92.

- González, M.T. (2005). Departamentos de Secundaria y desarrollo profesional. En M. Fernández Enguita y M. Gutiérrez Sastre (coords.), Organización escolar, profesión docente y entorno comunitario. Madrid: Akal, 229-254.

- Grossman, P.L. (1989) Un estudio comparado: Las fuentes del conocimiento didáctico del contenido en la enseñanza del inglés en Secundaria. Profesorado. Revista de Currículum y Formación del Profesorado, 9 (1), 2005.

- Grossman, P.L., Wilson, S.M. y Shulman, L.S. (1989) Profesores de sustancia: El conocimiento de la materia para la enseñanza. Profesorado. Revista de Currículum y Formación del Profesorado, 9 (2), 2005.

- Gudmundsdottir, S. (1998) La naturaleza narrativa del saber pedagógico sobre los contenidos. En H. McEwan y K. Egan (compls.), La narrativa en la enseñanza, el aprendizaje y la investigación. Buenos Aires: Amorrortu, 52-71.

- Gudmundsdottir S. y Shulman, L.S. (1990).El conocimiento didáctico en ciencias sociales. Profesorado. Revista de currículum y formación del profesorado, 9, 2 (2005) 37 Profesorado. Revista de Currículum y Formación del Profesorado, 9 (2), 2005.

- Klafki, W. (1986). Los fundamentos de una didáctica crítico-constructiva. Revista de Educación, 280 (mayo-agosto), 37-79.

- $\quad$ Liston, D.P. y Zeichner, K.M. (1997). Formación del profesorado y condiciones sociales de escolarización. Madrid: Morata.

- Marcelo, C. (1993). Cómo conocen los profesores la materia que enseñan. Algunas contribuciones de la investigación sobre Conocimiento Didáctico del Contenido. En L. Montero y J.M. Vez (eds.), Las didácticas específicas en la formación del Profesorado (I). Santiago de Compostela: Tórculo, 151-185.

- Marcelo, C. (1987b). Planificación y enseñanza, Sevilla, Servicio de Publicaciones de la Universidad.

- Marcelo, C. (1994). Formación del profesorado para el cambio educativo. Barcelona: PPU

- Montero, L. (2001). La construcción del conocimiento en la enseñanza. En C. Marcelo (ed.), La función docente. Madrid: Síntesis, 47-83.

- Montero L. y Vez, J.M. (eds.) (1993). Las didácticas específicas en la formación del Profesorado (I). Santiago de Compostela: Tórculo, 151-185. 
- Montero, L. y Vez, J.M. (2004). Didácticas específicas. En F. Salvador Mata, J.L. Rodríguez Diéguez y A. Bolívar (dirs.): Diccionario/Enciclopedia de Didáctica. Archidona (Málaga): Aljibe, vol. I, 427-448.

- $\quad$ Schwab, J.J. (1973). Problemas, tópicos y puntos en discusión. En S. Elam (Comp.), La educación y la estructura del conocimiento. Buenos Aires: El Ateneo, 1-37.

- Shulman, L.S. (1989). Paradigmas y programas de investigación en el estudio de la enseñanza: una perspectiva contemporánea. En M.C. Wittrock (ed.): La investigación de la enseñanza, I. Enfoques, teorías y métodos. Barcelona: Paidós/MEC, 9-91.

- Stodolsky, S.S. (1991). La importancia del contenido en la enseñanza: Actividades en las clases de matemáticas y ciencias sociales. Barcelona: Paidós/MEC.

- Tamir, P. (1991) Conocimiento profesional y personal de los profesores y de los formadores de profesores. Profesorado. Revista de Currículum y Formación del Profesorado, 9 (2), 2005. 